\title{
Endosonographie in der Diagnostik und im Staging des Bronchialkarzinoms
}

\author{
Endosonography in Diagnosis and Staging of Lung Cancer
}

Autoren

Institut

\author{
R. Eberhardt, F. J. F. Herth
}

Abteilung Innere Medizin-Pneumologie (Chefarzt Prof. Dr. med. Felix JF Herth), Thoraxklinik am Universitätsklinikum Heidelberg

\section{Bibliografie}

DOI $10.1055 / \mathrm{s}-2006-944268$ Pneumologie 2006; 60; 562-567

(c) Georg Thieme Verlag KG

Stuttgart · New York

ISSN 0934-8387

\section{Korrespondenzadresse}

Dr. med. Ralf Eberhardt

Abteilung Innere Medizin -

Pneumologie - Thoraxklinik am

Universitätsklinikum Heidelberg

Amalienstraße 5

69126 Heidelberg

ralf.eberhardt@thoraxklinik-hei-

delberg.de

www.thoraxklinik-heidel-

berg.de

Serienherausgeber

H. Worth, Fürth

T. Schaberg, Rotenburg

\section{Zusammenfassung}

Heutzutage stellt die Endosonographie in Form des endobronchialen (EBUS) und des endoösophagealen Ultraschalls (EUS) eine Routineanwendung bei der Endoskopie in vielen pneumologischen Zentren dar. Drei verschiedene Techniken, die die Diagnostik und das Staging bei Patienten mit einem Bronchialkarzinom verbessern können, stehen zur Verfügung. Die klinische Anwendbarkeit und der diagnostische Zugewinn wurden in einer Vielzahl von Studien im Vergleich zu radiologischen und anderen diagnostischen Verfahren nachgewiesen. Es ist daher zu erwarten, dass die Bedeutung der Endosonographie als diagnostisches Instrument in naher $\mathrm{Zu}$ kunft weiter zunimmt. Speziell die Methoden der EUS-Feinnadelaspiration (EUS-FNA) und/ oder der EBUS kontrollierten transbronchialen Nadelaspiration (EBUS-TBNA) erscheinen in der Lage, invasivere Techniken wie die Mediastinoskopie in der Evaluation von Patienten mit einem Bronchialkarzinom zu verdrängen.

\section{Einleitung}

Auf dem Gebiet der Pneumologie haben sich in den vergangenen Jahren drei endosonographische Verfahren etabliert und eine zunehmende Bedeutung in der Diagnostik und Therapieplanung des Bronchialkarzinoms erhalten. Neben dem endobronchialen Ultraschall (EBUS) mittels radiärer Minisonden stehen mittlerweile Punktionsendoskope mit longitudinalem Schallkopf sowohl für die endoösophageale als auch für die endobronchiale Anwendung zur Verfügung.

\section{Abstract}

Today endosonography in form of endobronchial (EBUS) and endoesophageal ultrasound (EUS) is a routine adjunct to endoscopy in many pulmonary centers. Three different techniques are available and can improve the diagnosing and staging in patients with lung cancer. The clinical application and the diagnostic benefit have been established in a lot of studies compared with conventional radiologic methods and other diagnostic procedures. The role of endosonography is expected to grow in the near future as an important tool. Especially the approaches with EUS-fine needle aspiration (EUS-FNA) and/or EBUS controlled transbronchial needle aspiration (EBUSTBNA) may be able to replace more invasive methods like mediastinoscopy for evaluating patients with lung cancer.

\section{EBUS mittels radiärer Minisonden}

Seit Anfang der 1990er-Jahre wurden miniaturisierte Ultraschallminisonden entwickelt, die aufgrund ihres verringerten Durchmessers von 2,5 $\mathrm{mm}$ über den Arbeitskanal eines flexiblen Bronchoskops (Arbeitskanal = 2,8 mm) angewendet werden können. Die Bildgebung und damit die Aussagekraft der Methode sind im Wesentlichen von der Ankopplung an die Bronchialwand, von der Bildauflösung und der Eindringtiefe des Ultraschalls abhängig. Die Ultraschallfrequenz 

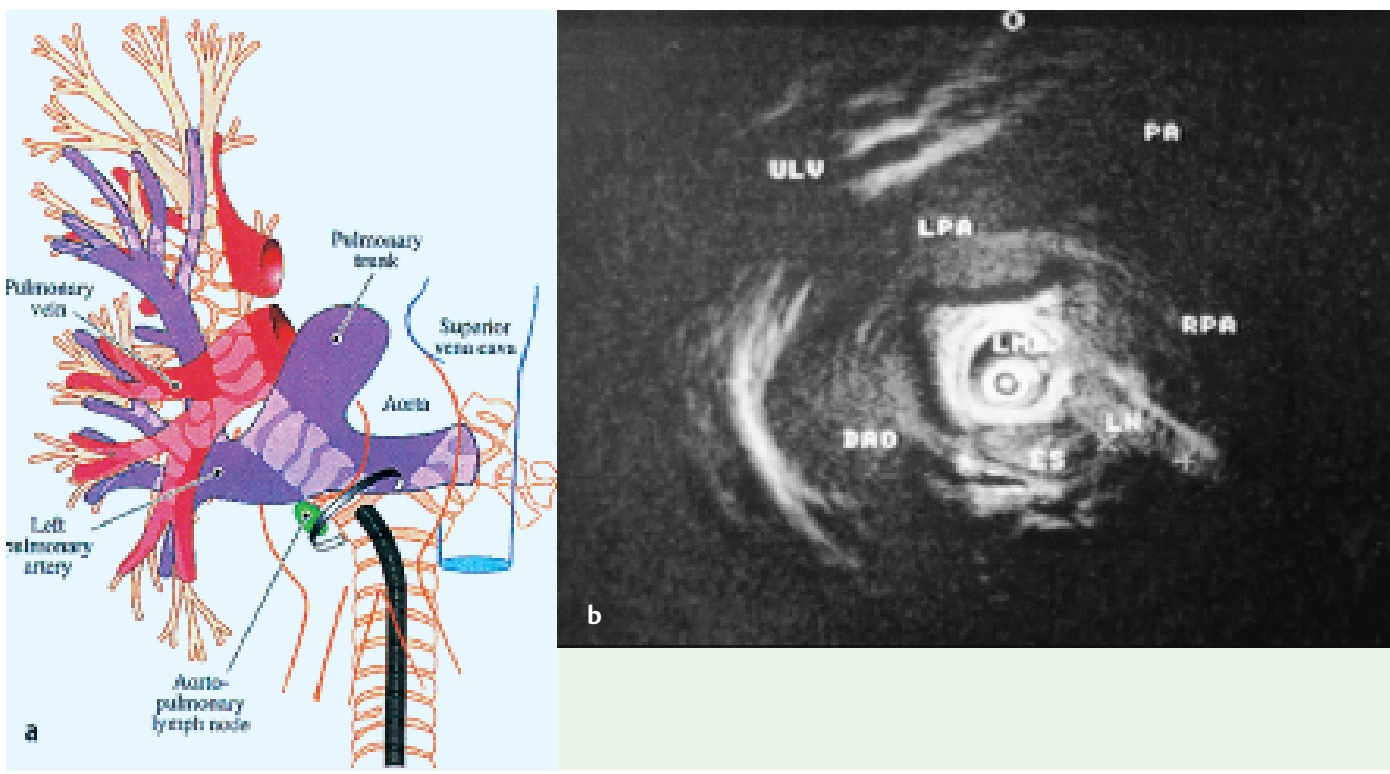

von $20 \mathrm{MHz}$ des rotierenden Transducers ermöglicht dabei eine hohe Nahauflösung, so dass eine gute Beurteilung der anliegenden bronchialen und parabronchialen Strukturen möglich ist. Aufgrund des Luftgehalts der zentralen Atemwege werden zur zirkulären Ankoppelung an die Bronchialwand die Sonden in einem flexiblen Katheter eingeführt, an dessen Spitze ein Ballon mit Wasser gefüllt werden kann. Das ist bei beidseitig belüfteter Lunge am lokal anästhesierten Patienten zentralwärts bis in die Hauptbronchien ohne Probleme möglich. Durch die Wasservorlaufstrecke kann dabei die Eindringtiefe auf $5 \mathrm{~cm}$ erhöht werden $[1,2]$.

Die sonographische Orientierung im Mediastinum ist schwierig. Das liegt neben der komplexen Anatomie und den Bewegungsartefakten durch Atmung und Pulsationen auch an den ungewohnten Bildschnitten, da die Sonden dem Verlauf der Atemwege folgen müssen. Für die räumliche Orientierung sind markante anatomische Strukturen und ihre Beziehung zueinander oft hilfreicher als die endoskopische Kontrolle der Sondenlage ( $\mathbf{A b b}$. 1). Die Lernkurve ist daher bei dieser Methode flach und die Aussagekraft Untersucher abhängig [3,4].
Abb. 1 a Anatomische Skizze und Darstellung der Schallebene

b Sonoanatomie des linken Hauptbronchus (LMB), der umgeben ist vom Ösophagus (ES), deszendierender Aorta (DAO) sowie linker (LPA) und rechter Pulmonalarterie (RPA), die aus dem Hauptstamm abzweigen (PA). Ventral der linken Pulmonalarterie erkennt man die Oberlappenvene (ULV), neben dem Ösophagus stellt sich ein kleiner Lymphknoten dar (LN). Ein Lymphknoten - wie in der Schemazeichnung grün abgebildet - ist nicht sichtbar.

\section{Endobronchialer Ultraschall EBUS-TBNA}

Eine Weiterentwicklung im Bereich des endobronchialen Ultraschalls stellt das seit 2004 allgemein verfügbare flexible Ultraschallbronchoskop mit integriertem 7,5-12 MHz Ultraschallkopf dar. Aufgrund eines separaten Arbeitskanals und einer 30 -Seitblickoptik kann die Nadel (22G) zur transbronchialen Nadelaspiration (TBNA) sowohl endoskopisch als auch sonographisch visualisiert werden. Damit werden in einer Untersuchung die endobronchiale Inspektion der Atemwege und die kontrollierte Punktion von parabronchialen und paratrachealen Strukturen unter Sicht möglich ( Abb.2). Ein Ballon an der Spitze des Endoskops kann die Ankopplung und die Bildgebung verbessern, ein Doppler-Modus erlaubt darüber hinaus, solide Strukturen sicher von zentralen Gefäßen zu unterscheiden.

Mit dem „EBUS-skop“ können sowohl mediastinale als auch hiläre Lymphknoten beidseits dargestellt und punktiert werden. Aufgrund der guten Kontrolle über die Nadelspitze ist mit dieser Methode auch eine sichere transbronchiale Punktion von Lymphknoten unter $1 \mathrm{~cm}$ möglich geworden [5-8].
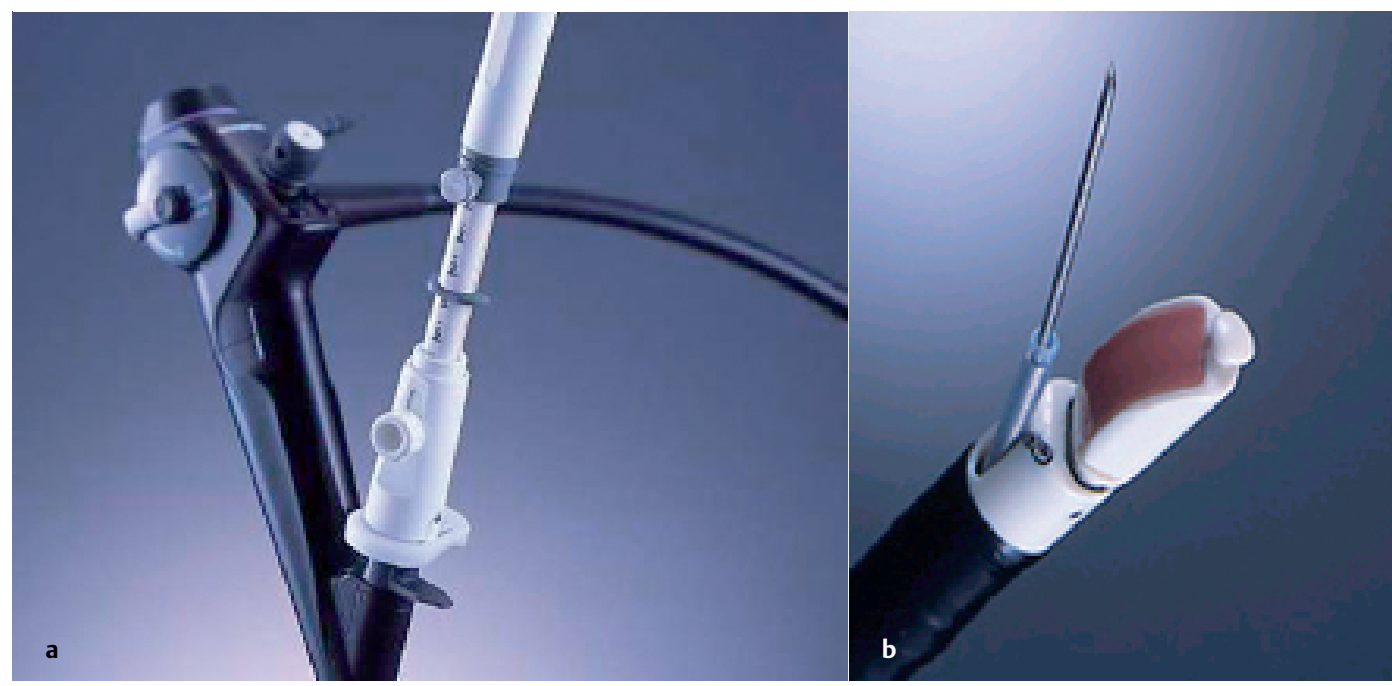

Abb. 2 Ultaschallbronchoskop (Olympus BFUC40F-OL5; Olympus Ltd., Tokyo, Japan): links mit am proximalen Ende fixierter 22G-Zytologienadel. Rechts distales Ende mit konvexem Schallkopf und ausgefahrener 22G-Zytologienadel. Die 30 -Seitblickoptik befindet sich zwischen Arbeitskanal und Schallkopf. 


\section{Endoösophagealer Ultraschall EUS-FNA}

Für den endoösophagealen Ultraschall (EUS) stehen Endoskope mit sowohl radiärer als longitudinaler Ultraschallausrichtung zur Verfügung. Für eine Zytologiegewinnung aus paraösophagealen Strukturen sind die radiären Sonden nicht geeignet. Daher kommen auch hier Punktionsendoskope mit einem 5-10 MHz-Longitudinal-Schallkopf und der Option der Feinnadelaspiration (EUS-FNA) zur Anwendung. Diese im Vergleich zum endobronchialen Ultraschall ältere Methode zur Dedektion und gezielten Feinnadelpunktion mediastinaler Läsionen ermöglicht jedoch keine Inspektion der Atemwege und nur eine eingeschränkte endoskopische Sicht im Bereich des Ösophagus. Aufgrund des Fehlens von endoösophagealen „Landmarken“ erfolgt hier die Orientierung über die Ultraschallanatomie $[9,10]$. Die Option eines mit Wasser füllbaren Ballons an der Endoskopspitze zur Verbesserung der sonographischen Bildqualität ist bei allen Geräten gegeben.

Der besondere Vorteil des EUS liegt im Zugriff auf das hintere und untere Mediastinum sowie auf Teile des aorto-pulmonalen Fensters [11]. Die Luft der Trachea und des rechten Hauptbronchus behindert jedoch die Darstellung der prätrachealen und rechts paratrachealen bzw. parabronchialen Lymphknotenstationen. Eine weitere Indikation für die EUS-FNA ist die gezielte transgastrale Untersuchung der Nebennieren bei Metastasenverdacht [12].

\section{Frühkarzinom}

Im Bemühen, die Prognose des Bronchialkarzinoms zu verbessern, wurde die Autofluoreszenzbronchoskopie (AFB) entwickelt, um bereits im Weißlicht noch nicht sichtbare maligne Läsionen der Schleimhaut zu erkennen. Da die Methode der Autofluoreszenz aber relativ unspezifisch ist, führt ihre Anwendung zu einer vermehrten Diagnose von benignen oder prämalignen Veränderungen. Durch den hochauflösenden EBUS mittels radiärer Minisonden lassen sich bis zu sieben Schichten der Tracheal- und Bronchialwand darstellen. In einer prospektiven Studie konnte dabei gezeigt werden, dass durch den zusätzlichen Einsatz des endobronchialen Ultraschalls die Vorhersage bezüglich Malignität in den Frühstadien verbessert werden kann. Die Korrelation zwischen der AFB und der Histologie konnte von 0,58 auf 0,92 gesteigert werden $[13,14](\bullet$ Abb. 3 ).

Die Diagnose Carcinoma in situ wird histologisch aufgrund der fehlenden Infiltration in die Lamina propria gestellt. Das Frühkarzinom hingegen ist durch die „fehlende radiologische Darstellbarkeit" definiert. Trotz dieses frühen Tumorstadiums muss aber das therapeutische Vorgehen differenziert werden. Eine japanische Arbeitsgruppe untersuchte 18 Patienten mit einem Frühkarzinom mittels radiärem endobronchialen Ultraschall. Bei neun Patienten zeigte sich das Tumorwachstum im EBUS auf die Bronchialwand beschränkt, während die andere Hälfte ein wandüberschreitendes Wachstum zeigte. Diese Patienten wurden chirurgisch oder in einem multimodalen Therapiekonzept behandelt. Die erste Gruppe erhielt hingegen ein rein endoskopisches Therapieverfahren in Form einer photodynamischen Therapie (PDT). Bei einem mittleren Beobachtungszeitraum von 24 Monaten zeigten letztere Patienten alle eine anhaltende komplette Remission [15]. Ein kurativer endoskopischer Therapieansatz des Frühkarzinoms ist damit in Abhängigkeit des endosonographischen Befundes gegeben.

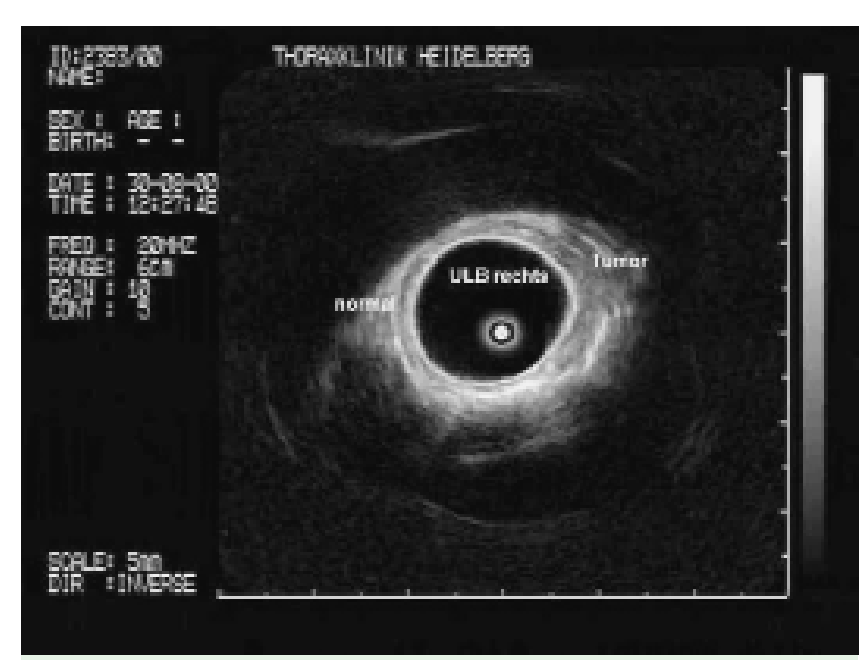

Abb. 3 Frühkarzinom (Tumor) am Abgang vom Segment S6 rechts. Rechts oben zeigt sich die verdickte Bronchialwand im Vergleich zu der noch erhaltenen normalen Bronchialwandung links (normal). Die EBUSMinisonde liegt im Unterlappenbronchus rechts (ULB).

\section{Lokales T-Staging}

In der Ausdehnung eines zentralen Tumorwachstums wird die radiologische Beurteilung mittels CT nur in ca. zwei Drittel der Fälle intraoperativ bestätigt $[16,17]$. In einer prospektiven Studie wurden Patienten mit einem zentralen Tumor sowohl mit einer Spiral-CT als auch mit endobronchialem Ultraschall untersucht und bezüglich Tumorinfiltration der Atemwege unabhängig klassifiziert. Dabei zeigte sich eine deutliche Überlegenheit des EBUS gegenüber dem bisherigen Goldstandard der CT (Sensitivität 89 vs. $25 \%$, Spezifität 100 vs. $80 \%$ ) [18].

Auch der endoösophageale Ultraschall ist aufgrund seiner besseren Bildauflösung der Computertomographie überlegen. In einer deutschen Arbeit wurden 97 von 201 Patienten mit einem zentralen Tumorwachstum und radiologischem Aortenkontakt operiert. Zuvor war die Frage der T4-Situation aufgrund einer Gefäßinfiltration sowohl mittels CT als auch endosonographisch beurteilt worden. Auch hier zeigt sich das sonographische Verfahren mit einer Sensitivität von $83 \%$ vs. $17 \%$ und einer Spezifität von $93 \%$ vs. $5 \%$ der Computertomographie überlegen [19].

\section{N-Staging}

Die Wertigkeit der transbronchialen Nadelaspiration (TBNA) für das regionale Lymphknotenstaging im Mediastinum ist belegt $[20,21]$. Durch die vorherige Darstellung der Lymphknoten durch EBUS ist eine Erhöhung der Trefferquote der sequentiell durchgeführten TBNA auf über $80 \%$ möglich $[22,23]$.

Seit kurzem steht die US-kontrollierte EBUS-TBNA zur Verfügung ( Abb.4). Nach bereits anfänglich guten Ergebnissen aus dem Jahre 2004 liegen jetzt die Daten einer internationalen Multicenterstudie mit 502 Patienten vor. Danach liegen die Sensitivität bei Lymphknoten mit einem mittleren Durchmesser von $1,6 \mathrm{~cm}$ bei $92 \%$, die Spezifität bei $100 \%$ und der positive Vorhersagewert bei $93 \%$. In allen Studien zur EBUS-TBNA wurde über keine schwereren Komplikationen berichtet [5,7,24].

Die transösophageale Endosonographie wird in der Pneumologie bereits seit 10 Jahren zur Diagnostik von mediastinalen 


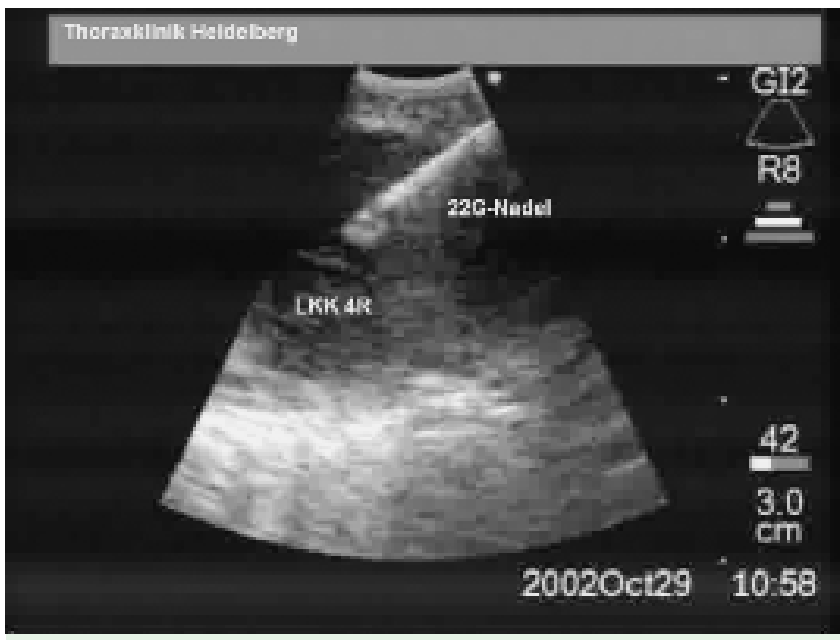

Abb. 4 Endosonographisches Bild eines vergrößerten Lymphknotens (LKK) in Position 4R. Man erkennt die Punktion mit einer 22-Gauge-Nadel von endobronchial.

Raumforderungen und zur Stadieneinteilung von Lungenkarzinomen angewendet. Durch die mittels endoösophageale Sonographie kontrollierte Punktion (EUS-FNA) lässt sich ebenfalls eine hohe Sensitivität von 80 bis zu $97 \%$ bei einer maximalen Spezifität von $100 \%$ in der Beurteilung vergrößerter Lymphknoten beim NSCLC erreichen [25-27]. In einer dänischen Arbeit wurde die EUS-FNA mit der Mediastinoskopie bezüglich Malignität von paratrachealen und subcarinalen Lymphknoten bei Patienten mit einem nicht-kleinzelligen Bronchialkarzinom verglichen. Dabei zeigt sich das minimal invasive endosonographische Verfahren dem chirurgischen Vorgehen überlegen [28].

Mit beiden endosonographischen Verfahren lassen sich auch Lymphknoten darstellen und punktieren, deren Größe weniger als $10 \mathrm{~mm}$ beträgt. Dabei kann man unabhängig vom Zugangsweg in ca. ${ }^{1} / 5$ der Patienten mit einem NSCLC eine fortgeschrittene Tumorerkrankung und/oder eine mediastinale Lymphknotenmetastasierung trotz unauffälliger CT nachweisen $[7,8,29,30]$.

Insgesamt drei Arbeiten haben das endobronchiale Verfahren mit der endoösophagealen Technik verglichen [12,31,32]. In allen Arbeiten zeigen die beiden Verfahren vergleichbare Trefferquoten. Dabei hängen die Ergebnisse von der anatomischen Erreichbarkeit der Lymphknotenstationen durch die unterschiedlichen Techniken ab. So zeigt sich der EBUS prätracheal sowie rechts paratracheal überlegen, während der EUS auch die distalen paraösophagealen Lymphknoten erreicht. Entsprechend lässt sich die diagnostische Aussagekraft in allen drei Arbeiten steigern, wenn man die beiden Verfahren kombiniert. Somit ergibt sich eine minimal-invasive Alternative zur Mediastinoskopie.

\section{M-Staging}

Eine Rolle in der Abklärung extrathorakaler Metastasen spielt nur der endoösophageale US mit der Option der Punktion der Nebennieren. Während das endosonographische Aufsuchen der rechten Nebenniere nicht in allen Fällen gelingt, ist die linke Nebenniere aufgrund ihrer Nähe zum Magen bei fast allen Patienten darstellbar. Somit gibt sich die Möglichkeit der transgastra- len Punktion der linken Nebenniere, während für die rechte häufig ein transabdominelles Verfahren zur Anwendung kommt. Die linke Nebenniere sollte immer im Rahmen einer EUS-Anwendung mituntersucht werden, da der Nachweis einer Nebennierenmetastase bei einem Bronchialkarzinom in bis zu 5\% der Fälle das therapeutische Vorgehen beeinflusst [11].

\section{Intrapulmonale Låsionen}

Die transbronchiale Biopsie pulmonaler Rundherde wird normalerweise unter Durchleuchtungskontrolle durchgeführt. Dabei ist die diagnostische Wertigkeit dieser Methode von der Größe und der Lokalisation der pulmonalen Läsionen abhängig. Durch den sonographischen Nachweis von solidem Gewebe in Abgrenzung zu belüftetem gesunden Lungengewebe lassen sich auch intrapulmonale Rundherde mittels EBUS lokalisieren (Abb. 5). Dabei ist die Trefferquote mittels EBUS mit der unter Durchleuchtung vergleichbar. Bei Läsionen kleiner als $3 \mathrm{~cm}$ konnte sogar ein - wenn auch nicht signifikanter - diagnostischer Zugewinn durch den endobronchialen Ultraschall (80 vs. $57 \%$ ) gezeigt werden $[33,34]$. Durch den Einsatz der radiären Minisonden lassen sich auch pulmonale Läsionen dedektieren und biopsieren, die unter Durchleuchtung nicht darstellbar sind [35].

\section{Diskussion}

Das Bronchialkarzinom ist die häufigste Krebstodesursache der westlichen Welt, nicht nur beim Mann, sondern auch mit zunehmender Häufigkeit bei der Frau [36]. Eine Diagnose kann dabei in der Mehrzahl der Fälle durch ein bronchoskopisches Biopsieverfahren gestellt oder bestätigt werden. Die Prognose, aber auch die Therapieentscheidung sind jedoch nicht nur von der Typisierung des Karzinoms, sondern auch von dessen Ausbreitung abhängig. Insbesondere beim nicht-kleinzelligen Bronchialkarzinom (NSCLC) entscheidet eine mediastinale Metastasierung oder Tumorinfiltration über eine eventuelle Inoperabilität oder ein multimodales Therapiekonzept. Somit wird eine genaue prätherapeutische Evaluation der mediastinalen Strukturen und Lymphknoten notwendig [37,38].

Der Blick des Bronchoskopeurs bleibt auf den endobronchialen Befund und die Mukosa der zentralen Atemwege beschränkt. Zwar können indirekte Zeichen wie pathologische Gefäßzeichnungen der Schleimhaut oder Kompression und Verlagerung der Bronchien Hinweise auf ein tumoröses Geschehen geben. Ein Großteil der malignen Prozesse erfasst aber die parabronchialen Strukturen, so dass ein Blick über die Bronchialwand hinaus notwendig wird [39]. Radiologische Verfahren wie die Computertomographie (CT) und die Magnetresonanztomographie (MRT) haben sich als zu unzuverlässig für eine exakte Stadieneinteilung des Bronchialkarzinoms erwiesen. Als radiologisches Kriterium für einen malignen Lymphknotenbefall gilt auch heute noch in der CT eine Größe von mehr als $10 \mathrm{~mm}$ in der kurzen Achse. Vergleicht man die Ergebnisse mit dem chirurgischen Staging, so ist die präoperative Stadieneinteilung nur in 50-60\% der Fälle korrekt [16,41-43]. Eine Positronenemissions-Tomographie (PET) kann die diagnostische Aussage verbessern. Dabei ist die Kombination als PET-CT den einzelnen Verfahren im Staging des NSCLC sogar noch überlegen. Doch selbst dann müssen PET-positive Lymphknoten aufgrund der zahlreichen falsch-positiven Befunde insbesondere in niedrigen 

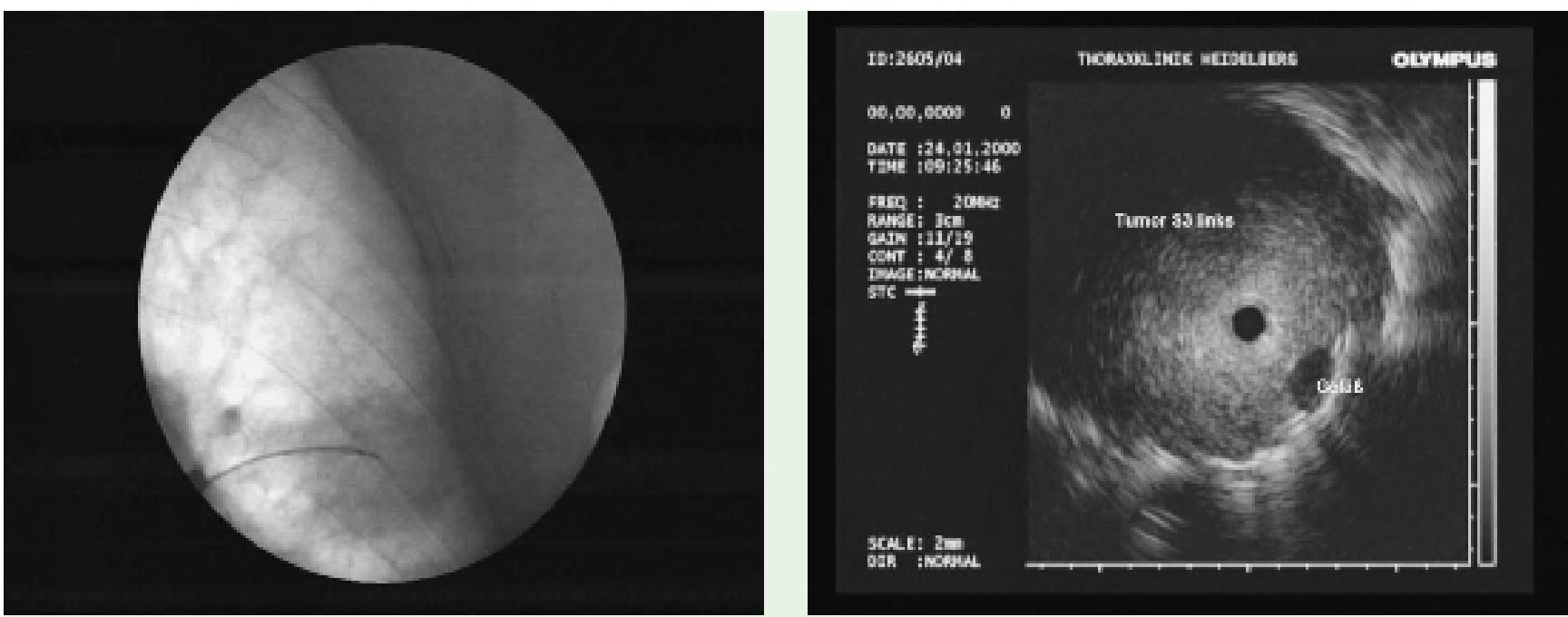

Abb. 5 NSCLC im Segment 3 des linken Oberlappens. Links die Darstellung unter Durchleuchtung, die EBUS-Sonde projiziert sich auf den Tumor. Rechts das korrespondierende Ultraschallbild mit einem großen soliden Tumor. Man erkennt ein von Tumor umgebenes Gefäß im rechten unteren Bildabschnitt.

Tumorstadien histologisch und/oder zytologisch geklärt werden $[44,45]$.

Mit der Endosonographie stehen heutzutage Techniken zur Verfügung, die die Diagnostik, aber auch die Stadieneinteilung des Bronchialkarzinoms verbessern. Dabei erlangen nicht nur die sonographische Darstellung der Tumorausdehnung wie z.B. beim Frühkarzinom, sondern auch der zytologische Nachweis einer mediastinalen oder adrenalen Metastasierung eine therapieentscheidende Bedeutung. Selbst kleinste Läsionen können sicher abgeklärt werden.

Insbesondere durch die Möglichkeit der minimal invasiven Punktion mediastinaler Lymphknoten unter endosonographischer Kontrolle treten die EBUS-TBNA und die EUS-FNA in Konkurrenz zur Mediastinoskopie. Dabei versprechen die endoskopischen Verfahren geringere Kosten bei minimalen Komplikationen. Der Nutzen ist mittlerweile durch zahlreiche prospektive Studien evaluiert und bestätigt. Inwieweit diese Tatsachen im Stagingalgorithmus bzw. im Behandlungskonzept des Bronchialkarzinoms zukünftig Berücksichtigung finden sollten, muss daher dringlich geklärt und definiert werden.

\section{Literatur}

1 Herth F, Becker HD. Endobronchial Ultrasound of the airways and the mediastinum. Monaldi Arch Chest Dis 2000; 55 (1): 36-45

2 Herth F, Becker HD. Endobronchial Ultrasound (EBUS) - assessment of a new diagnostic tool in bronchoscopy. Onkologie 2001; 24: 151 - 154

3 Hürther T, Hanrath P. Endobronchial sonography: feasibility and preliminary results. Thorax 1992; 47: 565-567

4 Falcone F, Fois F, Grosso D. Endobronchial Ultrasound. Respiration 2003; 70 (2): 179-194

5 Krasnik M, Vilman P, Larsen SS et al. Preliminary experience with a new method of endoscopic transbronchial real time ultrasound guided biopsy for diagnosis of mediastinal hilar lesions. Thorax 2003; 58: $1083-1086$

6 Herth FJF, Eberhardt $R$. The role of endobronchial ultrasound in diagnosis, staging and treatment. Therapy 2005; 2 (2): 223-228

7 Yasufuku K, Chiyo M, Sekine Y et al. Real-time endobronchialultrasound-guided transbronchial needle aspiration of mediastinal and hilar lymph nodes. Chest 2004; 126: $122-128$

8 Herth FJ, Ernst A, Eberhardt $R$ et al. Endobronchial ultrasound-guided transbronchial needle aspiration of lymph nodes in the radiologically normal mediastinum. Eur Respir J, 2006 [Epub ahead of print],
9 Annema JT, Veselic M, Rabe KF. Transösophageale Sonographie des Mediastinums. In: Mathis G (Hrsg.). Bildatlas zur Lungen- und Pleurasonographie. 3. Aufl. Berlin, Heidelberg, New York: Springer, 2001: 100112

10 Jacobson BC, Hirota WK, Goldstein JL et al. Amerivcan Society for Gastrointestinal Endoscopy. The role of EUS for evaluation of mediastinal adenopathy. Gastroentestinal Endoscopy 2004; 58: 819-821

11 Dietrich CF, Hocke M. Mediastinum. In: Dietrich CF (Hrsg.). Endoscopic Ultrasound. An Introduction. Stuttgart: Thieme, 2006: 323 - 333

12 Herth FJ, Lunn W, Eberhardt $R$ et al. Transbronchial versus transesophageal ultrasound-guided aspiration of enlarged mediastinal lymph nodes. Am J Respir Crit Care Med 2005; 171 (10): 1164 - 1677

13 Shirakawa T, Tanaka F, Becker HD. Layer structure of the central airways viewed using endobronchial ultrasonography (EBUS). In: Yoshimura H, Kida A, Arai T et al. (Hrsg.). Bronchology and Bronchoesophagology: stae of the art. Amsterdam: Elesevier, 2001: 921 - 923

14 Herth F, Becker HD, LoCicero J IIIrd et al. Endobronchial ultrasound is useful for further classification of suspicious lesions detected by autofluorescence-bronchoscopy. J Bronchol 2003; 10 (4): 249-252

15 Miyazu Y, Miyazawa T, Iwamoto $Y$ et al. Endobronchial ultrasonography in the assesment of centrally located early-stage lung cancer before photodynamic therapy. Am J Respir Crit Care Med 2002; 165 (6): $832-837$

16 Bonomo L, Ciccotosto C, Guidotto A et al. Lung cancer staging: the role of computer tomography and magnetic resonance imaging. Eur J Radiol 1996; 23: 35-45

17 Layer G, Kaick G van. Staging des nichtkleinzelligen Bronchialkarzinoms mit CT und MRT. Radiologie 1990; 30: 155 - 163

18 Herth FJ, Ernst A, Schulz M et al. Endobronchial Ultrasound reliably differentiates between airway infiltration and compression by tumor. Chest 2003; 123: $458-462$

19 Schroder C, Schönhofer B, Vogel B. Transesophageal echographic determination of aortic invasion by lung cancer. Chest 2005; 127 (2): 438 442

20 Metha AC, Kavuru MS, Meeker DP et al. Transbronchial needle aspiration for histology specimens. Chest 1989; 96: $1268-1272$

21 Gasparini S, Zuccatosta L, DeNictolis M. Transbronchial needle aspiration for mediastinal lesions. Monaldi Arch Chest Dis 2000; 55 (1): 2932

22 Herth FJ, Becker HD, Ernst A. Ultrasound-guided transbronchial needle aspiration: an experience in 242 patients. Chest 2003; 123: 604-607

23 Herth FJF, Ernst A, Becker HD. Conventional vs. endobronchial ultrasound guided transbronchial needle aspiration (TBNA) - a randomized trial. Chest 2004; 123: 458 - 462

24 Herth FJ, Eberhardt R, Vilmann P et al. Real-time, endobronchial ultrasound-guided, transbronchial needle aspiration: a new method for sampling mediastinal lymph nodes. Thorax, 2006, [Epub ahead of print] 
25 Caddy G, Conron M, Wright G et al. The accuracy of EUS-FNA in assessing mediastinal lymphandenopathy and staging patients with NSCLC. Eur Respir J 2005; 25 (3): 410-415

26 Fritscher-Ravens A, Sririam PV, Bobrowski C et al. Mediastinal lymphadenopathy in patients with or without previous malignancy: EUSFNA based differential cytodiagnosis in 153 patients. Am J Gastroenterol 2000; 95: 2278-2284

27 Annema JT, Hoekstra OS, Smit EF et al. Towards minimally invasive staging strategy in NSCLC: analysis of PET positive mediastinal lesions by EUS-FNA. Lung cancer 2004; 44: 53-60

28 Larsen SS, Vilmann P, Krasnik $M$ et al. Endoscopic ultrasound guided biopsy versus mediastinoscopy for analysis of paratracheal and subcarinal lymph nodes in lung cancer staging. Lung cancer 2005; 48 (1): $85-92$

29 LeBlanc JK, Devereaux BM, Imperiale TF et al. Endoscopic ultrasound in non-small cell lung cancer and negative mediastinum on computed tomography. Am J Respir Crit Care Med 2005; 171 (2): 177 - 182

30 Wallace MB, Ravenel J, Block MI et al. Endoscopic ultrasound in lung cancer patients with a normal mediastinum on computed tomography. Ann Thorac Surg 2004; 77 (5): 1763 - 1768

31 Rintoul RC, Skwarski KM, Murchison JT et al. Endobronchial and endoscopic ultrasound-guided real-time fine-needle aspiration for mediastinal staging. Eur Respir J 2005; 26 (1): 182 - 183

32 Vilmann P, Krasnik M, Larsen SS et al. Transesophageal endoscopic ultrasound-guided fine-needle aspiration (EUS-FNA) and endobronchial ultrasound-guided transbronchial needle aspiration (EBUS-TBNA) biopsy: a combined approach in the evaluation of mediastinal lesions. Endoscopy 2005; 37 (9): 833 - 839

33 Herth FJ, Ernst A, Becker HD. Endobronchial ultrasound-guided transbronchial lung biopsy in solitary pulmonary nodules and peripheral lesions. Eur Respir J 2002; 20 (4): 972 - 974
34 Omori S, Takiguchi Y, Hiroshima K et al. Peripheral pulmonary diseases: evaluation with endobronchial US initial experience. Radiology 2002; 224 (2): $603-608$

35 Herth FJ, Eberhardt R, Becker HD et al. Endobronchial ultrasoundguided transbronchial lung biopsy in fluoroscopically invisible solitary pulmonary nodules: a prospective trial. Chest 2006; 129 (1): 147 150

36 Nackaerts $K$, Axelson O, Branbilla E et al. Epidemiology of lung cancer: a general update. Eur Resp Rev 2002; 84: 112 - 121

37 Spira A, Ettinger DS. Multidisciplinary management of lung cancer. N Engl J Med 2004; 350: 379 - 392

38 Fritscher-Ravens A, Soehendra N, Schirrow L et al. Role of transesophageal endosonography-guided fine-needle aspiration in the diagnosis of lung cancer. Chest 2000; 117: $339-345$

39 Becker HD, Kayser K, Schulz V et al. Atlas of Bronchology. Technique, Diagnosis Differential Diagnosis, Therapy. Philadelphia, Hamilton: B. C. Decker, Inc, 1991

40 Sihoe AD, Yim AP. Lung cancer staging. J Surg Res 2004; 117: 92 - 106

41 Gdeedo A, Schil P van, Corhouts B et al. Prospective evaluation of computed tomography and mediastinoscopy in mediastinal lymph node staging. Eur Respir J 1997; 10: 1547 - 1551

42 Dillemans B, Deneffe G, Vershakelen J et al. Value of computed tomography and mediastinoscopy in preoperative evaluation of mediastinal nodes in non-small cell lung cancer: a study of 569 patients. Eur J Cardiothorac Surg 1994; 8: 37-42

43 Hintze C, Biederer J, Wenz HW et al. MRI in staging of lung cancer. Radiologe 2006; 46 (4): 251 - 259

44 Wahl RI, Quint LE, Grenough $R$ et al. Staging of mediastinal non-small cell lung cancer with FDG-PET, CT and fusion images: preliminary prospective evaluation. Radiology 1994; 191: 371 - 377

45 Cerfolio RJ, Ojha B, Bryant AS et al. The accuracy of integrated PET-CT compared with dedicated PET alone for the staging of patients with nonsmall cell lung cancer. Ann Thorac Surg 2004; 78: 1017 - 1023 